Draft Version July 24, 2020

Preprint typeset using $\mathrm{LATEX}_{\mathrm{E}}$ style emulateapj v. 01/23/15

\title{
ON THE POWER TO CONSTRAIN THE ACCRETION HISTORY OF MASSIVE BLACK HOLES VIA SPIN MEASUREMENTS BY UPCOMING X-RAY TELESCOPES
}

\author{
Xiaoxia Zhang ${ }^{1, \dagger}$, Youjun $\mathrm{Lu}^{2,3}$, Dandan Wang, ${ }^{4}$, and TaOtao Fang ${ }^{1}$ \\ ${ }^{1}$ Department of Astronomy, Xiamen University, Xiamen, Fujian 361005, China; ${ }^{\dagger}$ zhangxx@xmu.edu.cn; fangt@xmu.edu.cn \\ 2 National Astronomical Observatories, Chinese Academy of Sciences, Beijing 100012, China; luyj@nao.cas.cn \\ ${ }^{3}$ School of Astronomy and Space Sciences, University of Chinese Academy of Sciences, No. 19A Yuquan Road, Beijing 100049, China \\ ${ }^{4}$ Department of Astronomy, School of Physics and Astronomy, Shanghai Jiao Tong University, 800 Dongchuan Road, Shanghai 200240, \\ China \\ Draft version July 24, 2020
}

\begin{abstract}
The spin distribution of massive black holes (MBHs) contains rich information on their assembly history. However, only limited information can be extracted from currently available spin measurements of MBHs owing to the small sample size and large measurement uncertainties. Upcoming X-ray telescopes with improved spectral resolution and larger effective area are expected to provide new insights into the growth history of MBHs. Here we investigate, at a proof of concept level, how stringent constraints can be placed on the accretion history of MBHs by the spin measurements from future $\mathrm{X}$-ray missions. We assume a toy model consisting of a two-phase accretion history composed of an initial coherent phase with a constant disk orientation, followed by a chaotic phase with random disk orientations in each accretion episode. By utilizing mock spin data generated from such models and performing Bayesian Markov Chain Monte Carlo simulations, we find that most accretion models of MBHs can be reconstructed provided that $\gtrsim 100 \mathrm{MBH}$ spins are measured with an accuracy of $\lesssim 0.1$. We also quantify the precision of the reconstructed parameters by adopting various combinations of sample sizes and spin accuracies, and find that the sample size is more crucial to model reconstruction once the spin accuracy reaches $\sim 0.1$. To some extent, a better spin accuracy will compensate for a small sample size and vice versa. Future X-ray missions such as the Advanced Telescope for High Energy Astrophysics and the enhanced X-ray Timing and Polarimetry mission, may provide spin measurements of $\gtrsim 100 \mathrm{MBHs}$ with an uncertainty of $\sim 0.04-0.1$ and will thus put strong constraints on the MBH growth history.
\end{abstract}

Subject headings: accretion, accretion disks; black hole physics; galaxies: nuclei; X-rays: galaxy

\section{INTRODUCTION}

Massive black holes (MBHs) in galactic centers are expected to be rotating, as the acquisition of angular momenta accompanies the growth of mass either through $\mathrm{MBH}$ mergers or gas accretion. It has been revealed that MBHs gain their masses mainly through gas accretion during the quasi-stellar object/active galactic nucleus (AGN) phases (e.g., Yu \& Tremaine 2002; Marconi et al. 2004; Yu \& Lu 2004; Shankar et al. 2009) by comparing the local MBH mass density with the accreted mass density over the cosmic time via the Soltan (1982) argument. Accretion modes may leave imprints on the spin evolution of MBHs (e.g., King et al. 2008; Dotti et al. 2013; Volonteri et al. 2013), and observationally determined spin distribution will in turn provide insights into the accretion history of MBHs. Coherent accretion with almost fixed disk orientation will efficiently increase the $\mathrm{MBH}$ spin, to an extreme value when the mass is $\sim 2.4$ times larger than the initial mass (e.g., Thorne 1974). Instead, chaotic accretion composes a number of small episodes and in each episode the gas cloud infalls with a random direction of orbital angular momentum, and spins down the $\mathrm{MBH}$ because the radius of the innermost stable circular orbit (ISCO) is larger for a retrograde disk, thus the injection of negative angular momenta is more effective (e.g., Bardeen et al. 1972; Moderski et al. 1998).

Observationally, the spins of MBHs are mostly mea- sured by modeling the reflected X-ray spectra of AGNs with a basic assumption that the accretion disk is geometrically thin and optically thick (see Brenneman 2013; Revnolds 2014, 2019, for reviews of MBH spin measurements). In this case, the ISCO radius, which is crucial to the degree of relativistic effects on the X-ray spectrum in terms of broadened and skewed line profiles (e.g., Fabian et al. 1989; Laor 1991), is solely determined by the MBH spin (Bardeen et al. 1972), and the modeling of the profiles will in turn provide a measure of the MBH spin (e.g., Tanaka et al. 1995). Currently, MBHs with relatively robust spin measurements amount to no more than $\sim 30$, most of which are rapidly rotating Brenneman 2013; Revnolds 2014; Vasudevan et al. 2016, see also Zhang \& Lu 2019).

Despite the limited size of the spin sample, current data do provide some clues to the growth history of MBHs (e.g., Dotti et al. 2013; Sesana et al. 2014; Li et al. 2015). In our previous work (Zhang \& Lu 2019), we adopted a two-phase accretion model with an initial coherent phase followed by a chaotic one, and obtained constraints on the $\mathrm{MBH}$ accretion history by comparing the spin-mass distributions resulting from various models with that of the observed samples via the twodimensional KolmogorovâĂŞSmirnov (2D-KS) test. We found that chaotic accretion is necessary and the disk-to$\mathrm{BH}$ mass ratio in each episode is about $1 \%-2 \%$. By its nature, 2D-KS tests do not allow us to constrain mod- 
els with more than two free parameters, something that one can do within the Bayesian framework. However, the current sample size, in addition to the error budget, imposes limits on performing concrete Bayesian method.

Future X-ray telescopes such as Advanced Telescope for High ENergy Astrophysics (Athena; Barret et al. 2018) and the Lynx X-ray Observatory (Lynx; Bandler et al. 2019; Gaskin et al.|2019) will make significant improvements to the spectral resolution (to $2-3 \mathrm{eV}$ at $\sim 6 \mathrm{keV}$ ), and missions like the enhanced X-ray Timing and Polarimetry mission (eXTP; Zhang et al. 2019), Spectroscopic Time-Resolving Observatory for Broadband Energy X-rays (STROBE-X; Rav et al. 2019), and the High-Energy X-ray Probe (HEX-P; Madsen et al. 2018) will provide wide effective areas (up to $5 \mathrm{~m}^{2}$ at $\sim 6 \mathrm{keV}$ ) with a broadband energy coverage. Those will greatly enlarge the current spin sample and improve the accuracy of spin measurements, and will set unprecedented constraints on the assembly history of MBHs. In this paper, we make use of mock spin samples generated from two-phase accretion models, to investigate how well the growth history of MBHs can be constrained with those future X-ray detectors.

The paper is organized as follows. In Section 2, we describe the accretion model and equations governing the mass and spin evolution of MBHs. The mock samples generated from various models are presented in Section 3, followed by a description of Bayesian statistics in Section 4 Reconstruction results on various models are given in Section 5 , by adopting samples of different sizes and spin accuracies. We discuss our results in Section 6 and present our conclusions in Section 7 .

\section{ACCRETION MODEL AND EVOLUTION OF MBH SPINS}

The current spin sample of MBHs exhibits a trend in which lighter black holes (BHs) mostly rotate faster and heavier ones rotate more slowly, indicating that chaotic accretion may play a critical role in shaping the evolution of MBHs. We therefore follow Zhang \& Lu (2019) and consider accretion history models composed of both coherent and chaotic accretion, which may be taken as an approximation to the true accretion history (e.g., Model A with three parameters described below). Note that the real accretion history of the MBHs may depend on detailed properties of their host galaxies and could be different among individual MBHs. For simplicity, here we only consider the dependence on the mass of the host galaxy and thus the MBH mass, since the MBH mass correlates well with the stellar mass of the host galaxy (e.g., Häring \& Rix 2004; Hopkins et al. 2007). Our model could be approximated as the mean of the assembly history of those MBHs with similar host properties (stellar mass, velocity dispersion, etc.)

Initially, the host galaxy is probably rich in gas and the fueling of the central MBH is continuous and coherent, at a sub- or super-Eddington rate. With the consumption of gas, the MBH may later enter into a sub-Eddington chaotic accretion phase, composed of many small accretion episodes with random disk orientations. The mass of the gas cloud accreted in each chaotic episode is assumed to follow a power-law dependence on the $\mathrm{MBH}$ mass $M_{\bullet}$, i.e.,

$$
M_{\mathrm{cl}}\left(M_{\bullet}\right)=b M_{\bullet}\left(\frac{M_{\bullet}}{10^{8} M_{\odot}}\right)^{\gamma},
$$

where $b$ and $\gamma$ are constant parameters. If $\gamma=0$, then the mass of the gas cloud scales linearly with the MBH mass; if $\gamma=-1$, then the cloud mass is a constant for each episode in the chaotic accretion phase. The whole cloud is assumed to form an accretion disk with negligible mass loss. For super-Eddington accretion, the accretion disk is thick in geometry, while for a sub-Eddington rate, it forms a standard thin disk instead (Shakura \& Sunyaev 1973; Novikov \& Thorne 1973).

The division of the two phases is characterized by parameter $f_{\mathrm{c}}$ such that the second phase starts once the $\mathrm{MBH}$ mass reaches a factor $f_{\mathrm{c}}$ of the final mass $M_{\bullet}, \mathrm{f}$. If $f_{\mathrm{c}}=1$, then the MBH only experiences coherent accretion; if $f_{\mathrm{c}} \leq M_{\bullet, i} / M_{\bullet}$,f with $M_{\bullet}, i$ the initial $\mathrm{MBH}$ mass, then only chaotic accretion happens. $f_{\mathrm{c}}$ could be a constant, but it could also be dependent on the $\mathrm{MBH}$ final mass (e.g., see Dotti et al. 2013; Sesana et al. 2014; Zhang et al. 2019). Here the final mass means the mass of a quiescent $\mathrm{MBH}$ at the present time $(z=0)$. The dependence of $f_{\mathrm{c}}$ is implied by MBHs with different final masses having different accretion histories. Below we consider three models.

Model A: $f_{\mathrm{c}}$ is a constant $(\leq 1) . \quad b$ and $\gamma$ are also constant parameters (same for the following two models).

Model B: $f_{\mathrm{c}}$ follows a power-law dependence on the $\mathrm{MBH}$ mass, i.e.,

$$
f_{\mathrm{c}}\left(M_{\bullet}\right)=f_{0}\left(\frac{M_{\bullet}}{10^{8} M_{\odot}}\right)^{\alpha},
$$

where $f_{0}$ and $\alpha$ are both constant parameters and $M_{\bullet}, i M_{\bullet}$ f $\leq f_{\mathrm{c}} \leq 1$. If $\alpha=0$, then it reduces to Model A.

Model C: $f_{\mathrm{c}}$ also depends on the MBH mass but it follows a hyperbolic-tangent law such that $f_{\mathrm{c}}=1$ for MBHs with $M_{\bullet}>M_{1}$ and $f_{\mathrm{c}}=0$ for $M_{\bullet}<M_{2}$, where $M_{1}>M_{2}$, i.e., (see Gallo \& Sesana 2019, for similar expressions)

$$
f_{\mathrm{c}}\left(M_{\bullet}\right)=0.5+0.5 \tanh \left(2.5^{\beta} \log \frac{M_{\bullet}}{M_{0}}\right),
$$

where $\beta$ and $M_{0}$ are parameters. $M_{0}$ marks the mass at which the MBH has $f_{\mathrm{c}}=0.5$, and for convenience we introduce $m_{0} \equiv \log \left(M_{0} / M_{\odot}\right) ; \beta$ determines the slope of the transition from $f_{\mathrm{c}}=0$ at the low-mass end to $f_{\mathrm{c}}=1$ at the high-mass end, and the transition is faster for a larger $\beta$.

Regardless of the disk geometry, the mass growth of an MBH is completely determined by the accretion rate $\dot{M}$ and the specific energy $E_{\text {in }}$ at the inner disk boundary $R_{\text {in }}$ if the kinetic energy loss is negligible, i.e.,

$$
\frac{d M_{\bullet}}{d t}=f_{\mathrm{Edd}} \frac{E_{\text {in }}}{1-E_{\text {in }}} \frac{M_{\bullet}}{t_{\text {Edd }}},
$$

where $f_{\text {Edd }}$ is the Eddington ratio, and $t_{\text {Edd }}=4.5 \times 10^{8} \mathrm{yr}$ is the Eddington timescale. The inner boundary of the disk is different for thick and thin disks. For thin disks, $R_{\text {in }}$ is the ISCO radius, which is a monotonically determined by the $\mathrm{MBH}$ spin, i.e., $R_{\mathrm{in}}=3+Z_{2} \mp\left[\left(3-Z_{1}\right)(3+\right.$ $\left.\left.Z_{1}+2 Z_{2}\right)\right]^{1 / 2}$, where $Z_{1}$ and $Z_{2}$ are functions of spin, and the upper and lower cases of ' $\mp$ ' are respectively for prograde and retrograde orbits (Bardeen et al. 1972). For thick disks, $R_{\text {in }}$ is between the marginally bound and 
marginally stable orbits (Kozłowski et al. 1978). Below we only consider accretion rate capped by the Eddington limit and thus a thin-disk in geometry. As the accretion rate mainly affects the accretion time instead of the spin-mass evolutionary tracks, this will have little effect on our main results. With the assumption that the Eddington ratio is logarithmically dependent on the accretion rate, $R_{\text {in }}$ can be derived through interpolation (for details see Zhang \& Lu 2019). At a given spin, the specific energy $E_{\text {in }}$ (and also the specific angular momentum $\Phi_{\text {in }}$ in Equation (5) ) at any $R_{\text {in }}$ can be obtained (Bardeen et al. 1972). The mass-to-energy conversion efficiency $\eta$ is related to $E_{\text {in }}$ as $\eta=1-E_{\text {in }}$ if the kinetic energy loss is neglected.

For the general case of an inclined accretion disk with respect to the $\mathrm{MBH}$ spin, owing to the frame-dragging effect, the inner disk will be bent to the equatorial plane of the $\mathrm{MBH}$ and the outer disk maintains the original orientation (Bardeen \& Petterson 1975). Then the evolution of $\mathrm{MBH}$ angular momentum vector $\mathbf{J}$ • is governed by

$$
\frac{d \mathbf{J} \bullet}{d t}=\dot{M} \frac{G M \bullet}{c} \Phi_{\text {in }} \hat{\mathbf{l}}+\frac{4 \pi G}{c^{2}} \int_{\text {disk }} \frac{\mathbf{L} \times \mathbf{J} \bullet}{R^{2}} d R,
$$

where $\Phi_{\text {in }}$ is the specific angular momentum at $R_{\text {in }}, \hat{\mathbf{l}}$ is a unit vector paralleled with $\mathbf{J}_{\bullet}$, and $\mathbf{L}$ is the angular momentum vector of the disk per unit area. The first term on the right side denotes the angular momentum change due to plunge-in of material at $R_{\text {in }}$, and only leads to the modification of the spin module, while the second term describes the gravito-magnetic interaction between the disk and $\mathrm{MBH}$, and only causes spin direction change. Equations (4) and (5) are solved via the adiabatic approximation, i.e., the disk transits through a sequence of steady warped states over time interval $\delta t \ll t_{\mathrm{al}}$, where $t_{\mathrm{al}}$ is the alignment timescale (Perego et al. 2009). If the disk and $\mathrm{MBH}$ angular momenta already align with each other, then the spin direction does not change for the rest of the episode, and Equation (5) reduces to

$$
\frac{d a}{d t}=\left(\Phi_{\text {in }}-2 a E_{\text {in }}\right) \frac{f_{\mathrm{Edd}}}{\left(1-E_{\mathrm{in}}\right) t_{\mathrm{Edd}}},
$$

where $a$ is the dimensionless spin parameter defined as $|a|=c\left|\mathbf{J}_{\bullet}\right| /\left(G M_{\bullet}^{2}\right)$, and $a$ is positive (negative) if the disk is corotating (counterrotating) around the $\mathrm{MBH}$.

In our calculation, the initial mass of MBHs is fixed at $10^{5} M_{\odot}$, the final mass is randomly selected over $10^{6} M_{\odot}$ and $10^{10} M_{\odot}$ in logarithmic space, and the initial spin is randomly drawn from a uniform distribution between 0 and 1 . The accretion rate of the initial coherent phase is fixed at $\dot{m} \equiv \dot{M} / \dot{M}_{\mathrm{Edd}}=0.3$ where $\dot{M}_{\mathrm{Edd}}=16 L_{\mathrm{Edd}} / c^{2}$ with $L_{\text {Edd }}$ the Eddington luminosity, and the Eddington ratio $f_{\text {Edd }}$ in each chaotic phase is set to be a constant that is randomly drawn from a Gaussian distribution with a mean of 0.1 and standard deviation of 0.3 in logarithmic space. The maximum spin is assumed to be the canonical value of 0.998 , i.e., $|a| \leq 0.998$ (Thorne 1974)

We first consider the best-fit model implied by our previous work, i.e., Model A with $\left(b, \gamma, f_{\mathrm{c}}\right)=$ $(0.012,0.00,0.46)$, and examples of spin evolution of MBHs are shown in the top panel of Figure 1. As demonstrated in Zhang \& Lu (2019), the initial sharp increase

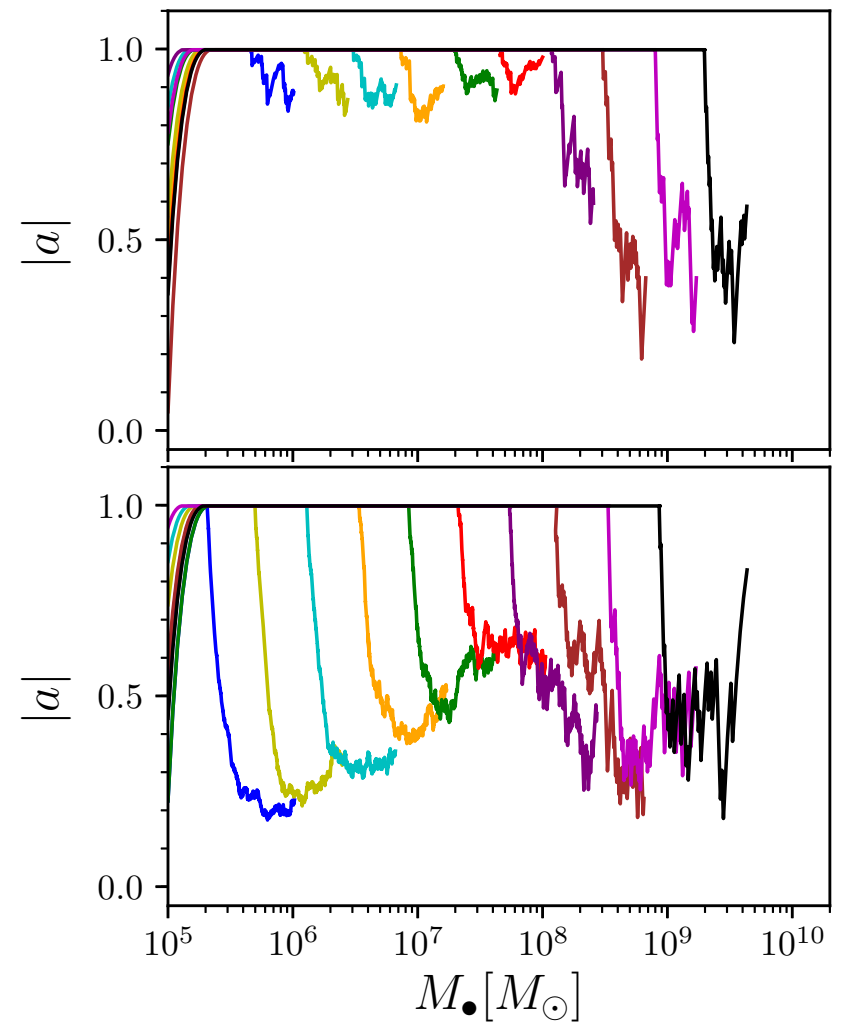

FIG. 1. - Examples of spin evolutionary curves for MBHs undergoing two-phase accretion with constant $f_{\mathrm{c}}$ and linearly scaled $M_{\mathrm{cl}}$ with the MBH mass, i.e., $\left(b, \gamma, f_{\mathrm{c}}\right)=(0.012,0.00,0.46)$ for the upper panel, and $\left(b, \gamma, f_{\mathrm{c}}\right)=(0.005,0.50,0.20)$ for the lower panel. Each of the MBHs has a fixed initial mass of $10^{5} M_{\odot}$ and a random initial spin over 0 and 1. Different colors represent $\mathrm{MBHs}$ with different final masses.

of the spin before the mass doubles is due to quick alignment of MBH spin to the disk angular momentum. Then the spin maintains the maximum value of 0.998 until the second chaotic phase decreases it somehow. More massive MBHs can be spun down to lower values because disk-to-BH angular momenta ratio decreases with increasing $\mathrm{MBH}$ mass as $J_{\mathrm{d}} / J_{\bullet} \propto M_{\bullet}^{-12 / 25}$. For arbitrary initial configurations, the criterion for final antialignment, i.e., $\cos \beta<-J_{\mathrm{d}} / J_{\text {• }}$ with $\beta$ being the initial angle between the two angular momenta King et al. 2005), is more frequently satisfied for high-mass BHs, and the probability is $\sim 0.5$ if $J_{\mathrm{d}} / J_{\bullet} \ll 1$.

We also consider alternative values of the parameters in Model A, i.e., $\left(b, \gamma, f_{\mathrm{c}}\right)=(0.005,0.50,0.20)$, which means that the second chaotic phase is more significant to the $\mathrm{MBH}$ growth, and that $J_{\mathrm{d}} / J_{\bullet}$ in each chaotic episode is smaller for low-mass BHs and larger for high-mass ones, compared to the former case. The spin evolution is shown in the lower panel of Figure 1, and the evolution trend is easy to understand. A smaller cloud and thus

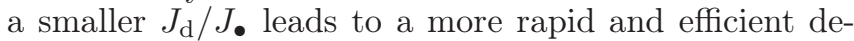
cline in the $\mathrm{MBH}$ spin, and a combination with a smaller $f_{\mathrm{c}}$ leads to the low spins for low-mass BHs. For highmass BHs, their lower spins are results of competition between a larger cloud and a more significant chaotic phase. This results in a feature in the spin-mass distribution in which low-mass and high-mass BHs have low spins while intermediate-mass BHs have relatively high spins (see also the lower panel of Figure 3). 


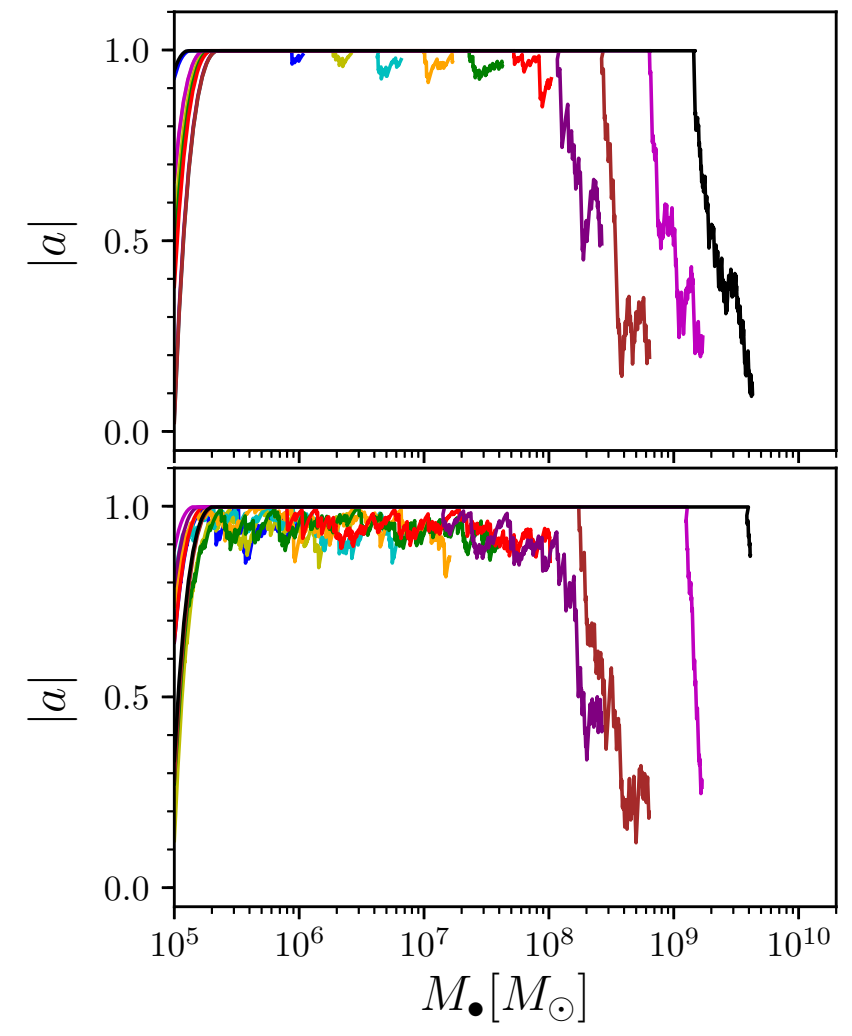

FIG. 2.- Examples of spin evolution for MBHs experiencing two-phase accretion model with power-law dependence of $f_{\mathrm{c}}$ on the MBH mass, i.e., $\left(b, \gamma, f_{0}, \alpha\right)=(0.010,-0.20,0.50,-0.10)$ for Model B (upper panel), and with hyperbolic-tangent form of $f_{\mathrm{c}}$ with $\left(b, \gamma, m_{0}, \beta\right)=(0.010,-0.20,9.00,1.00)$ for Model $\mathrm{C}$ (lower panel). The legend is the same as that in Figure 1

We then consider Model $\mathrm{B}$ and set $\left(b, \gamma, f_{0}, \alpha\right)=$ $(0.010,-0.20,0.50,-0.10)$. This results in the spin evolution of MBHs presented in the upper panel of Figure2 The spin decrease in the chaotic phase for $\mathrm{MBH}$ s with final masses $<10^{8} M_{\odot}$ is not as significant as that in the top panel of Figure 1 because of a larger $M_{\mathrm{cl}}$ and thus $J_{\mathrm{d}} / J_{\bullet}$ in each episode, while the spin can decrease to lower values for $>10^{8} M_{\odot}$ MBHs owing to a smaller $M_{\mathrm{cl}}$. The lower panel of Figure 2 is for Model C with $f_{\mathrm{c}}$ with $\left(b, \gamma, m_{0}, \beta\right)=(0.010,-0.20,9.00,1.00)$. Now lowmass BHs $\left(<10^{8} M_{\odot}\right)$ are not that extremely rotating, with $|a| \gtrsim 0.8$ for most of their lifetimes. This occurs because low-mass BHs experience pure chaotic accretion with $f_{\mathrm{c}} \sim 0$. The most massive BHs undergo a brief chaotic phase, and the spin remains at a high value (see the rightmost black curve).

\section{MOCK DATA}

Precise measurement of $\mathrm{MBH}$ spin via X-ray reflection spectroscopy relies on both the spectral resolution around $6 \mathrm{keV}$ to resolve the profile of the $\mathrm{Fe} \mathrm{K} \alpha$ line and high sensitivity in the $10-100 \mathrm{keV}$ band to characterize the reflection spectrum (e.g., García 2019). Future missions are expected to make improvements in both the sample size and accuracy of spin measurements (Miller 2007). For instance, the X-ray Integral Field Unit (X-IFU) instrument on board Athena could reach a mean spin measurement error of $\sim 0.04$ (Barret \& Cappi 2019). With a similar spectral energy resolution, Lynx may also achieve similar precisions. The eXTP incorpo-

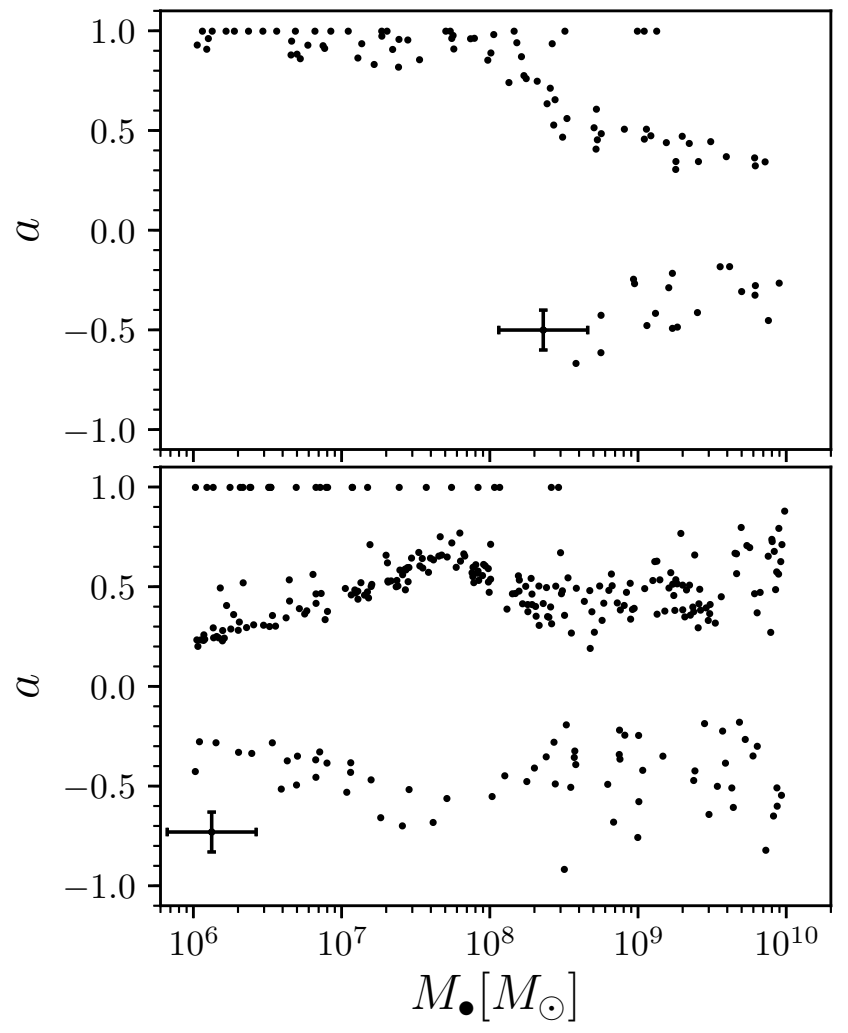

FIG. 3.- Spin-mass distribution of the mock sample generated from Model A with $\left(b, \gamma, f_{\mathrm{c}}\right)=(0.012,0.00,0.46)$ (upper panel) and $\left(b, \gamma, f_{\mathrm{c}}\right)=(0.005,0.50,0.20)$ (lower panel), including 100 and 300 objects, respectively. The error bar in each panel is to indicate the assumed measurement error for the whole sample, i.e., 0.1 for spin and 0.3 dex for mass.

rates the large area detector and the spectroscopic focusing array, and will make it possible to carry out detailed broad Fe line modeling on a sample of $\sim 400$ AGNs at different redshifts to measure $\mathrm{MBH}$ spins with an accuracy of $10 \%-20 \%$ (e.g., De Rosa et al. 2019). The concept mission STROBE-X has an effective area of $\sim 5 \mathrm{~m}^{2}$ at the iron line, which will lead to an accuracy of $<10 \%$ for spin measurement (Ray et al. 2019). In a word, at least hundreds of $\mathrm{MBH}$ spins are expected to be measured with an accuracy of $\lesssim 0.1$ in the future. We therefore consider samples including $100-1000 \mathrm{MBHs}$ with spin errors of $0.04-0.1$. The mock spin samples are generated from the accretion model described in Section 2.

We follow the mass and spin evolutionary tracks for a population of $1000 \mathrm{MBHs}$ according to the settings described in Section 2. Those spin-mass evolutionary curves are treated as templates, from which we randomly select a number of mock MBHs with known masses and spins as the mock data. We additionally assign a measurement error of 0.3 dex to the $\mathrm{MBH}$ mass according to the uncertainty of virial mass estimates (e.g., Shen et al. 2008, 2011). The accuracy of spin measurement could be different among various future X-ray detectors, and may also depend on the intrinsic spin value. Here we consider two cases of spin measurement errors, i.e., 0.1 and 0.04 .

The upper panel of Figure 3 shows the spin-mass distribution of 100 mock objects generated from Model A with $\left(b, \gamma, f_{\mathrm{c}}\right)=(0.012,0.00,0.46)$, which exhibits high spins $(|a| \gtrsim 0.8)$ for low-mass BHs and a broad range of spins $(|a| \sim 0.2-1)$ for high-mass ones. This is a natural 


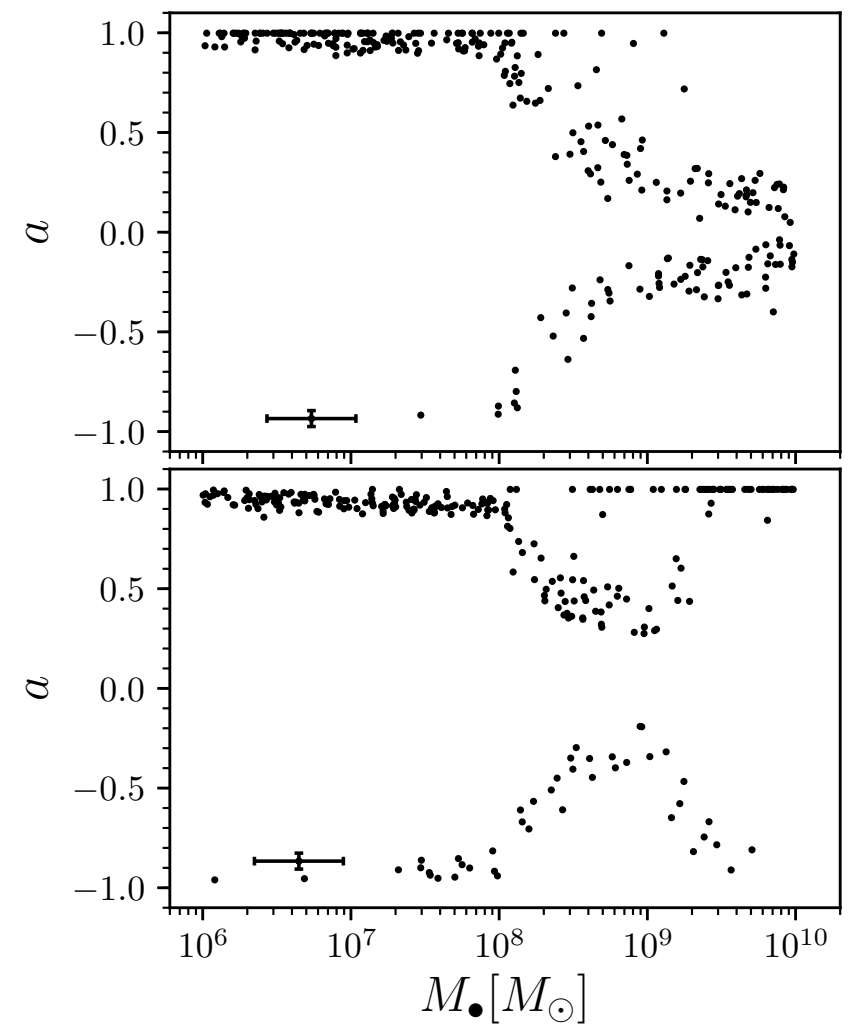

FIG. 4.- Spin-mass distribution of the sample generated from Model $\mathrm{B}$ with $\left(b, \gamma, f_{0}, \alpha\right)=(0.010,-0.20,0.50,-0.10)$ (upper panel), and from Model $\mathrm{C}$ with $\left(b, \gamma, m_{0}, \beta\right)=$ $(0.010,-0.20,9.00,1.00)$. The two sets of samples both include 300 objects with an assumed spin measurement error of 0.04 .

result of the spin evolutionary curves shown in the upper panel of Figure 1. There is also a fraction of counterrotating $\mathrm{BHs}$, and the fraction is higher for heavier ones, because a more massive $\mathrm{BH}$ has a higher probability of reaching a final anti-alignment configuration owing to a smaller disk-to-BH momenta ratio (see also the caption of Figure 1).

The spin distribution of 300 sources in the bottom panel of Figure 3 is Model A with $\left(b, \gamma, f_{\mathrm{c}}\right)=$ $(0.005,0.50,0.20)$, which mainly concentrates at intermediate spins $(|a| \sim 0.2-0.7)$. The distribution also follows the spin evolutionary tracks shown in the lower panel of Figure 1. A larger fraction of negative spins is found for low-mass BHs compared to those in the top panel, which is attributed to smaller $J_{\mathrm{d}} / J_{\bullet}$, thus there is higher probability of reaching an anti-alignment configuration.

For models with mass-dependent $f_{\mathrm{c}}$, the upper panel of Figure 4 shows a sample of size 100 generated from Model B with $\left(b, \gamma, f_{0}, \alpha\right)=(0.010,-0.20,0.50,-0.10)$. The lower panel is for Model $\mathrm{C}$ with $\left(b, \gamma, m_{0}, \beta\right)=$ $(0.010,-0.20,9.00,1.00)$, including 300 sources. The spin distributions of those samples simply follow the spin evolutionary tracks in Figure 2, with a fraction of negative spins depending on disk-to-BH angular momenta ratios and their initial configurations.

\section{BAYESIAN RECONSTRUCTION OF MBH ACCRETION HISTORY}

With the mock sample of MBH spin measurement generated in Section 3, the parameters associated with the accretion model are reconstructed by performing the
Bayesian Markov Chain Monte Carlo (MCMC) simulation. The model parameters are denoted as $\boldsymbol{\theta}$, and $D$ represents the mock data we generated in Section 3

The observations are assumed to be uncorrelated, therefore the probability of measuring $n_{i}$ objects in a particular $i$ th bin $\Delta M_{\bullet} \Delta a$ follows a Poisson distribution, i.e., (see also Sesana et al. 2011, 2014),

$$
p\left(n_{i}\right)=\frac{\lambda_{i}^{n_{i}} e^{-\lambda_{i}}}{n_{i} !},
$$

where $\lambda_{i}$ is the events number in the $i$ th bin expected by the model. If we divide the parameter space (spin vs. mass) into $K$ bins, then samples in the $i$ th bin can be easily counted. To further consider measurement errors of MBH mass and spin, we assume Gaussian distributions for $\log M_{\bullet}$ and $a$ around the mean values, with standard deviations of the assumed measurement errors. The probability distribution of spin is cut off at $a= \pm 0.998$ and normalized to 1 . The overall likelihood $p(D \mid \boldsymbol{\theta})$ of seeing these data under parameters $\boldsymbol{\theta}$ is the product of the Poisson probabilities, i.e.,

$$
p(D \mid \boldsymbol{\theta})=\prod_{i=1}^{K} \frac{\left(\lambda_{i}\right)^{n_{i}} e^{-\lambda_{i}}}{n_{i} !} .
$$

The logarithmic form is more frequently used, i.e.,

$$
\ln p(D \mid \boldsymbol{\theta})=\sum_{i=1}^{K} n_{i} \ln \left(\lambda_{i}\right)-\lambda_{i}-\ln \left(n_{i} !\right)
$$

With the consideration of measurement errors, $n_{i}$ may not be an integer, and the factorial cannot be computed. However, we note that the last term $-\ln \left(n_{i} !\right)$ is independent of models, and can therefore be neglected. According to Bayesian inference, the posterior distribution of $\boldsymbol{\theta}$ can be inferred through the likelihood and prior, i.e.,

$$
\begin{gathered}
p(\boldsymbol{\theta} \mid D) \propto p(D \mid \boldsymbol{\theta}) p(\boldsymbol{\theta}) . \\
\text { 5. RESULTS }
\end{gathered}
$$

\subsection{Reconstruction of the Three-parameter Model}

As for the reconstruction of accretion models, we first adopt the mock data produced from Model A with $\left(b, \gamma, f_{\mathrm{c}}\right)=(0.012,0.00,0.46)$ presented in the upper panel of Figure 3. The model parameters $\boldsymbol{\theta}=\left(b, \gamma, f_{\mathrm{c}}\right)$ are then set free, and we solve the equations governing the mass and spin evolution and randomly select 300,000 mock objects. During this process, the accretion rate $\dot{m}$ in the coherent phase is randomly chosen between 0.1 and 1 to make the thin-disk accretion model valid. This choice is to make sure that spin measurements for $\mathrm{MBH}$ are possible for this phase. The Eddington ratio in the chaotic phase is assumed to be moderate, i.e., $0.01 \leq f_{\mathrm{Edd}} \leq 1$ and the distribution is uniform in the logarithmic space over this range. For each set of parameters, we select 300,000 mock objects and compare the spin-mass distribution of those objects and the mock sample presented in Section 3 via the Bayesian technique.

Note here that the accretion history model we adopted to fit the data is the same model that generates the mock spin sample, in order to demonstrate that the accretion history can be well reconstructed. In reality, however, only one among a number of possible models that can 
best match the observational data is the best approximation to the true but unknown prior accretion history of MBHs. In Section 6.1 we will further investigate that whether a mock spin sample generated by one accretion history model can also be fit by a different one in order to show whether different accretion history models can be well distinguished from each other via the resulting spin distributions.

Figure 5 shows constraints on the model parameters, by adopting a sample consisting of $100 \mathrm{MBHs}$ with a spin measurement error of 0.1 (upper panel of Figure 3). The model parameters are well reproduced by comparing the $1 \sigma$ region (dashed lines) and the input values (cyan lines) shown in the figure. The posterior distributions of the model parameters are mostly Gaussian, except for $f_{c}$, which has a tail on the left side. This occurs because pure chaotic accretion $\left(f_{\mathrm{c}} \sim 0\right)$ can also result in an intermediate-to-high spin distribution, as demonstrated in Zhang \& Lu (2019). A larger sample may be required to remove the tail.

We therefore apply a sample with 300 mock objects in the the lower panel of Figure 3, which also has a spin accuracy of 0.1 , generated from Model A with $\left(b, \gamma, f_{\mathrm{c}}\right)=$ $(0.005,0.50,0.20)$. The model is reconstructed in Figure 6. Now there is no tail for the probability distribution of $f_{\mathrm{c}}$, and the parameters are confined in a narrower region compared to Figure 5. Our result implies that with a sample of size $\sim 300$ and spin error of $\sim 0.1$, the simple three-parameter model can be perfectly reconstructed, with the $b$ parameter best constrained with an accuracy of 0.0002 .

\subsection{Reconstruction of Four-parameter Models}

The results in Section 5.1 indicate that Model A with constant $f_{\mathrm{c}}$ can be very well reproduced provided that a sample of $300 \mathrm{MBH}$ spins is measured with an error of 0.1 . Here we relax $f_{\mathrm{c}}$ to vary among MBHs of different masses, and consider Model B with power-law dependence $f_{\mathrm{c}}$. The mock sample adopted is that shown in the upper panel of Figure 4, with an assumed spin measurement error of 0.04. The Bayesian constraint on the model parameters $\boldsymbol{\theta}=\left(b, \gamma, f_{0}, \alpha\right)$ is shown in Figure 7 .

As can be seen, constraints on the model parameters are not that stringent, compared to the case of Model A, despite the fact that more accurate spin data are applied. The posterior distributions of parameters $f_{0}$ and $\alpha$ cover broad regions and the input values of all the parameters are marginally consistent with the corresponding $1 \sigma$ boundary of the distribution. This might be because the sample is generated from the model with weak dependence of $f_{\mathrm{c}}$ on the MBH mass, i.e., $f_{\mathrm{c}} \propto M_{\bullet}^{-0.1}$, which is set to make sure that $f_{\mathrm{c}}$ does not exceed unity. The small difference of $f_{\mathrm{c}}$ among the different MBHs results in a bad constraint on $f_{\mathrm{c}}$ (in terms of $f_{0}$ and $\alpha$ ), which in turn causes a less stringent constraint on $M_{\mathrm{cl}}$ (in terms of $b$ and $\gamma$ ) because of degeneracy between $M_{\mathrm{cl}}$ and $f_{\mathrm{c}}$.

We therefore consider Model $\mathrm{C}$ with hyperbolictangent form of $f_{\mathrm{c}}$. By adopting the sample with 300 mock objects shown in the lower panel of Figure 4 the model parameters $\boldsymbol{\theta}=\left(b, \gamma, m_{0}, \beta\right)$ are constrained, as shown in Figure 8. The model parameters are now confined in a much narrower region as compared to the case of Model B shown in Figure 7. In addition, the input values of the model parameters are well within the $1 \sigma$ confidence regions, suggesting a successful reconstruction of the accretion model.

\subsection{The Role of Sample Size and Spin Accuracy}

There is great interest in exploring the full space of the sample size and spin accuracy. Here we simply consider two cases of spin accuracy, i.e., 0.1 and 0.04 , which can be approximated as the capacity of eXTP and Athena, respectively. For each of the two cases, we generate samples including 100, 300, and 1,000 MBHs from Model C. Similarly, we are able to obtain the posterior distributions of the model parameters.

A comparison of the reconstructed results with the input values of the model parameters is summarized in Table 1, which also includes results from Models A and B. For most of the cases listed there, the $1-\sigma$ confidence regions of the MCMC sampling are in line with the input values of the parameters. This is more readily satisfied if the mock sample applied has a smaller size and lower spin accuracy because the posterior distribution of the model parameter covers a broader range. An unexpected case is that when a sample of 1000 objects is applied to Model $\mathrm{C}$, the reconstructed $\beta$ deviates more from the mean value despite the fact that the standard deviation is smaller toward a larger sample size and better spin accuracy. One reason may be that the resulting spin distribution is still not sensitive enough to parameter $\beta$, compared to the other three parameters, which is implied by their error bars for cases with source numbers of 100 and 300. Other factors such as degeneracy between parameters and Poisson statistics may also contribute to this result. Our results indicate that a larger sample and better spin accuracy in the future will call for improvement in the accretion model of MBHs.

In addition to enabling comparison of the mean with the input value, $1-\sigma$ error is another quantity to measure the reconstruction of the accretion model. The standard deviations of the posterior distribution are treated as the constraint accuracy of the parameter, and its dependence on the sample size and spin precision are shown in Figure 9. The constraint accuracies of the parameters are mostly improved, as expected, with increasing object numbers of the mock sample and with improving accuracy of spin measurements. Constraints on the parameter $M_{0}$ (lower left panel) seem to not be sensitive to the spin accuracy, indicating that with a spin accuracy of 0.1 , parameter $M_{0}$ is already well reconstructed. This is determined by its intrinsic properties because the resulting spin distribution is quite sensitive to $\beta$ and it converges fast in the MCMC sampling. In the parameter space explored here, a larger sample size will somehow compensate for a lower spin accuracy, and vice versa.

\section{DISCUSSION}

\subsection{Fitting Mock with a Model Different from the Input One}

The results presented in Section 5 are obtained through MCMC simulations by adopting an accretion history model that is the same as the input model to generate the mock spin samples. However, the real accretion history is not known a priori and it can only be approximated via simplified assumptions on its form (e.g., Model A, B, or $\mathrm{C}$ ) and constrained by matching the model with observations. In the above section, we have demonstrated 


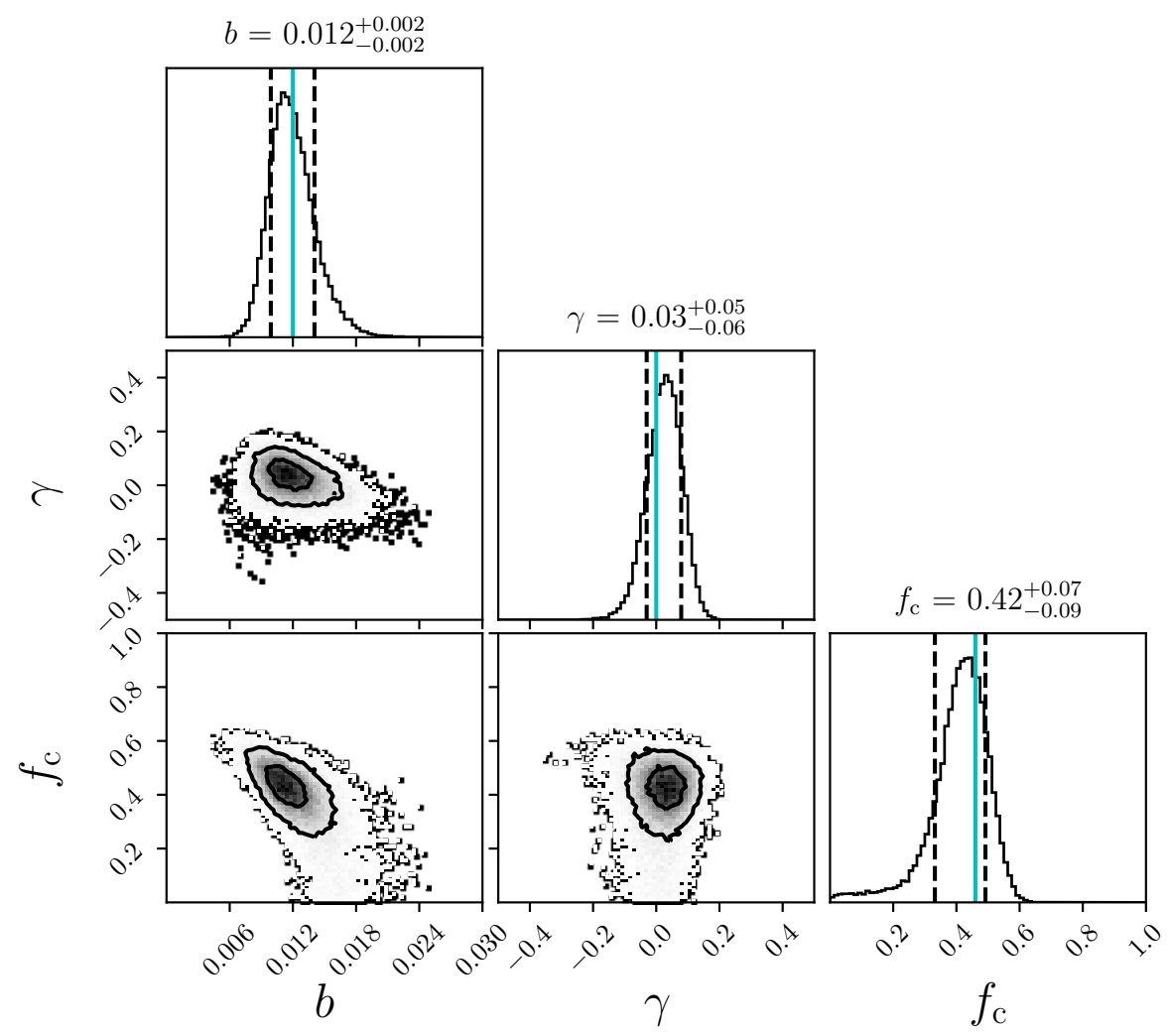

Fig. 5.- Joint and posterior distributions of the parameters involved in Model A. The sample adopted (shown in the upper panel of Figure 3 is generated from the model with $\left(b, \gamma, f_{\mathrm{c}}\right)=(0.012,0.00,0.46)$ (vertical cyan lines), including 100 sources with an assumed spin measurement error of 0.1 . The contours show $68 \%$ and $95 \%$ confidence levels, and each pair of dashed lines encloses a $1 \sigma$ region of the parameter.

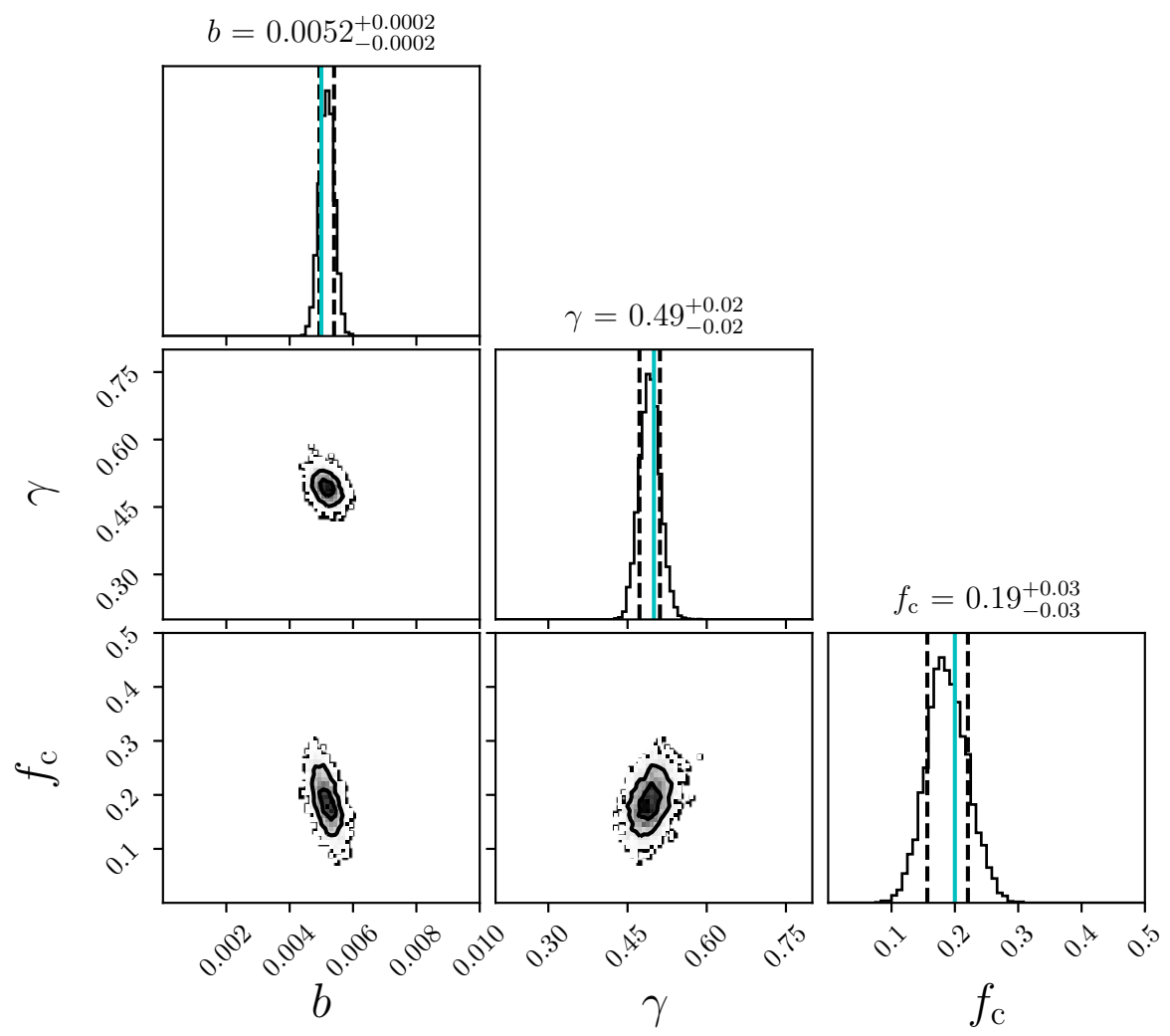

Fig. 6. - Same as Figure 5 but the sample applied (shown in the lower panel of Figure 3) contains $300 \mathrm{MBHs}$, generated from Model A with $\left(b, \gamma, f_{\mathrm{c}}\right)=(0.005,0.50,0.20)$ (cyan lines). 


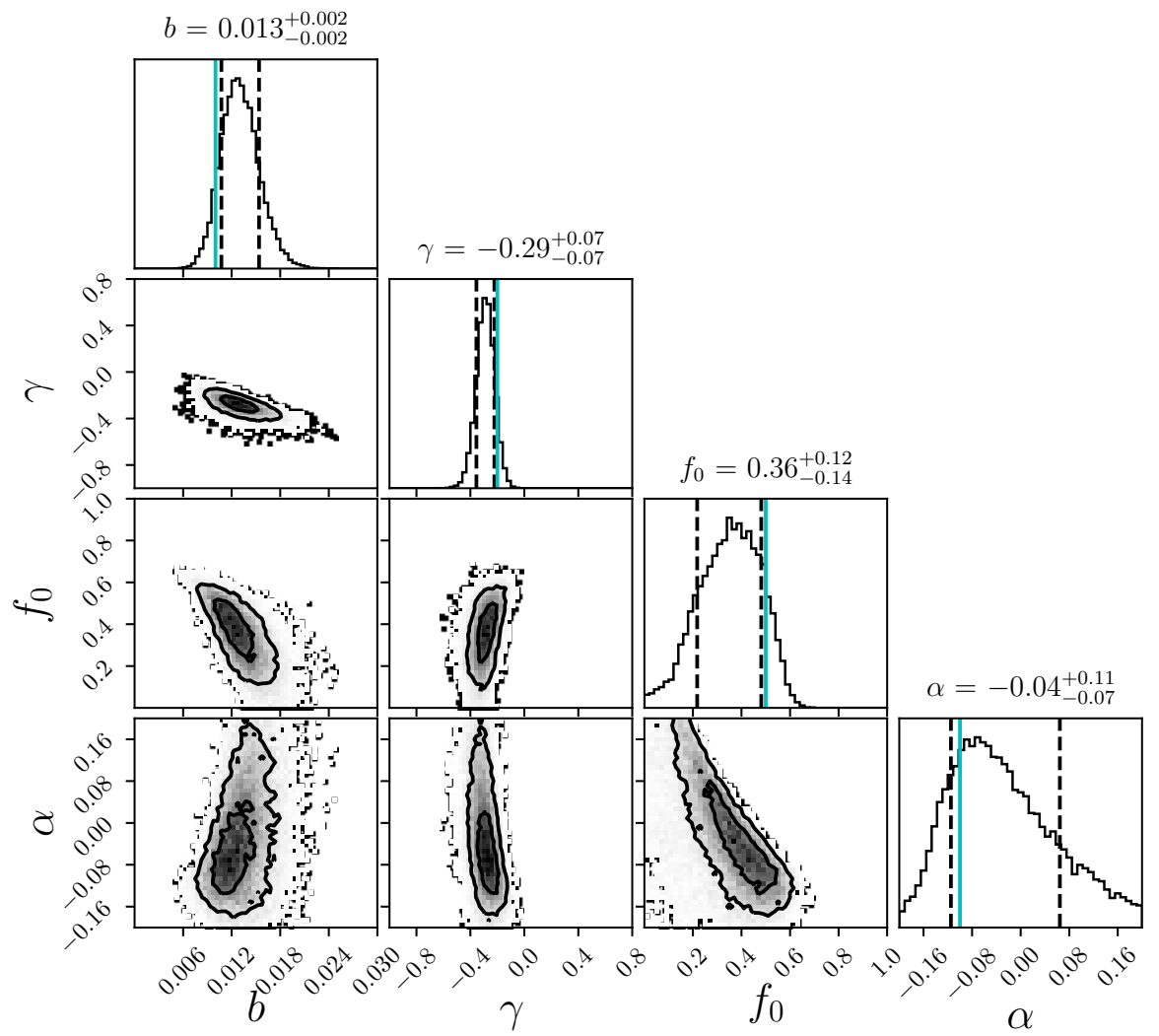

FIg. 7. - Joint and posterior distributions of the parameters involved in the accretion Model B. The sample adopted includes 300 sources with assumed spin measurement error of 0.04 (upper panel of Figure 4), generated from the model with $\left(b, \gamma, f_{0}, \alpha\right)=$ $(0.010,-0.20,0.50,-0.10)$ (cyan lines).

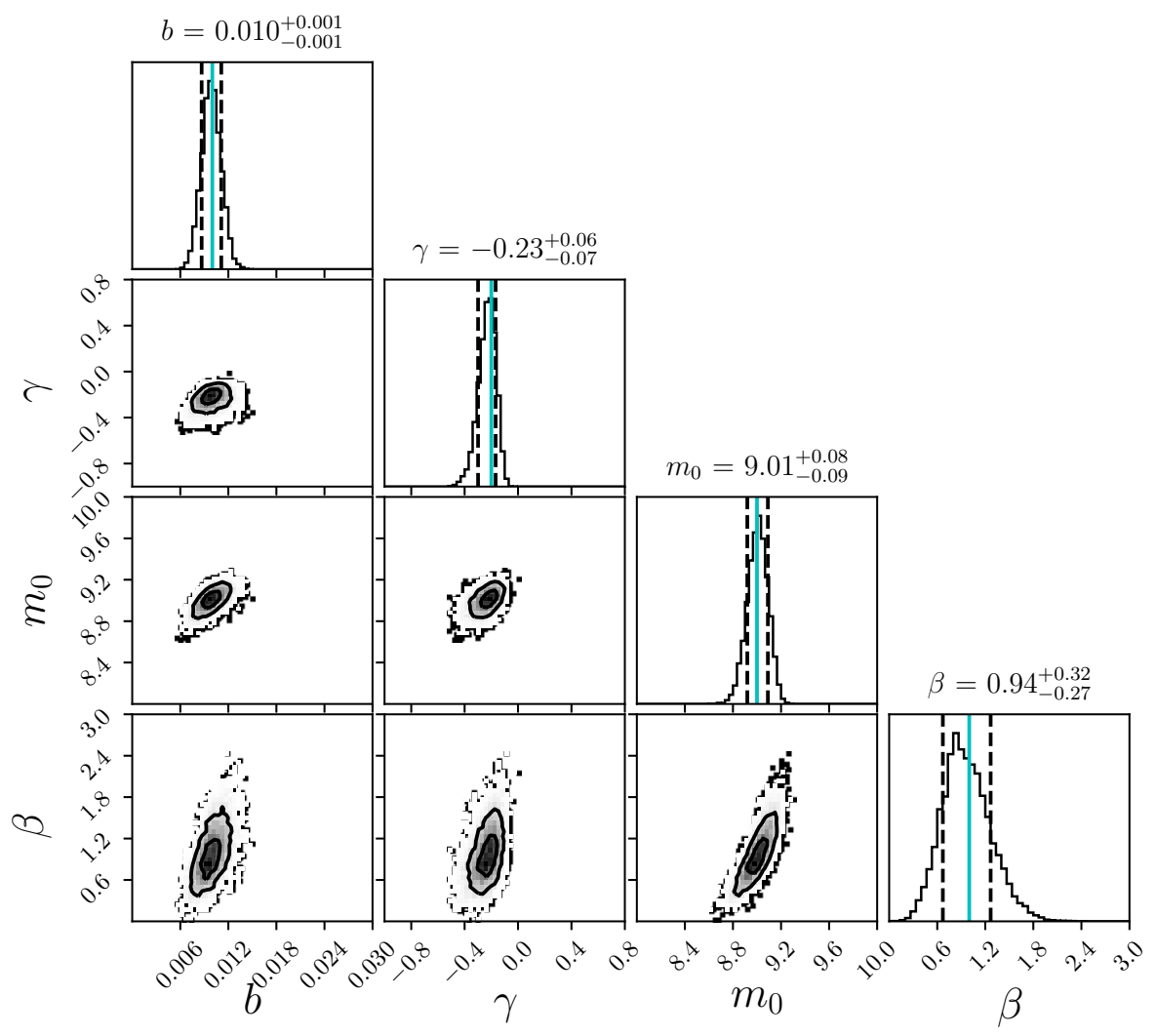

FIG. 8. - Joint and posterior distributions of the parameters involved in the accretion Model C. The sample adopted includes 300 sources with an assumed spin measurement errors of 0.04 (lower panel of Figure 4), generated from the model with $\left(b, \gamma, m_{0}, \beta\right)=$ $(0.010,-0.20,9.00,1.00)$ (cyan lines). 
TABLE 1

Comparison of Reconstructed Results and Input Values of the Parameters Associated with Different Versions of the Two-phase Accretion Model, by Utilizing Mock Samples of Various Sizes and Spin Accuracies.

\begin{tabular}{ccccc}
\hline \hline Model: Parameters & Number & Spin Error & Input Values & Reconstruction Results \\
\hline A: $\left(b, \gamma, f_{\mathrm{c}}\right)$ & 100 & 0.1 & $(0.012,0.00,0.46)$ & $\left(0.012_{-0.002}^{+0.002}, 0.03_{-0.06}^{+0.05}, 0.42_{-0.09}^{+0.07}\right)$ \\
& 300 & 0.1 & $(0.0050,0.50,0.20)$ & $\left(0.0052_{-0.0002}^{+0.0002}, 0.49_{-0.02}^{+0.02}, 0.19_{-0.03}^{+0.03}\right)$ \\
\hline B: $\left(b, \gamma, f_{0}, \alpha\right)$ & 300 & 0.04 & $(0.010,-0.20,0.50,-0.10)$ & $\left(0.013_{-0.002}^{+0.002},-0.29_{-0.07}^{+0.07}, 0.36_{-0.14}^{+0.12},-0.04_{-0.07}^{+0.11}\right)$ \\
\hline & 100 & 0.1 & & $\left(0.010_{-0.002}^{+0.002},-0.09_{-0.11}^{+0.08}, 9.06_{-0.15}^{+0.13}, 0.94_{-0.60}^{+0.71}\right)$ \\
& 100 & 0.04 & & $\left(0.010_{-0.002}^{+0.002},-0.16_{-0.08}^{+0.08}, 9.06_{-0.15}^{+0.13}, 0.96_{-0.43}^{+0.54}\right)$ \\
$\mathrm{C}:\left(b, \gamma, m_{0}, \beta\right)$ & 300 & 0.1 & $(0.010,-0.20,9.00,1.00)$ & $\left(0.010_{-0.001}^{+0.002},-0.22_{-0.11}^{+0.08}, 9.04_{-0.10}^{+0.09}, 1.16_{-0.39}^{+0.45}\right)$ \\
& 300 & 0.04 & & $\left(0.010_{-0.001}^{+0.001},-0.23_{-0.07}^{+0.06}, 9.01_{-0.09}^{+0.08}, 0.94_{-0.27}^{+0.32}\right)$ \\
& 1,000 & 0.1 & & $\left(0.0109_{-0.0007}^{+0.0008},-0.19_{-0.04}^{+0.04}, 9.09_{-0.05}^{+0.05}, 1.47_{-0.31}^{+0.34}\right)$ \\
& 1,000 & 0.04 & & $\left(0.0107_{-0.0006}^{+0.0006},-0.19_{-0.03}^{+0.03}, 9.08_{-0.05}^{+0.04}, 1.40_{-0.21}^{+0.23}\right)$ \\
\hline
\end{tabular}

Note. - Columns, from left to right: (1) the accretion model and associated parameters; (2) the number of objects in the mock sample; (3) assumed spin measurement error for each object in the mock sample; (4) input values of the model parameters to generate the mock sample; (5) Bayesian reconstruction results for the model parameters in terms of the mean value and $1 \sigma$ uncertainties.
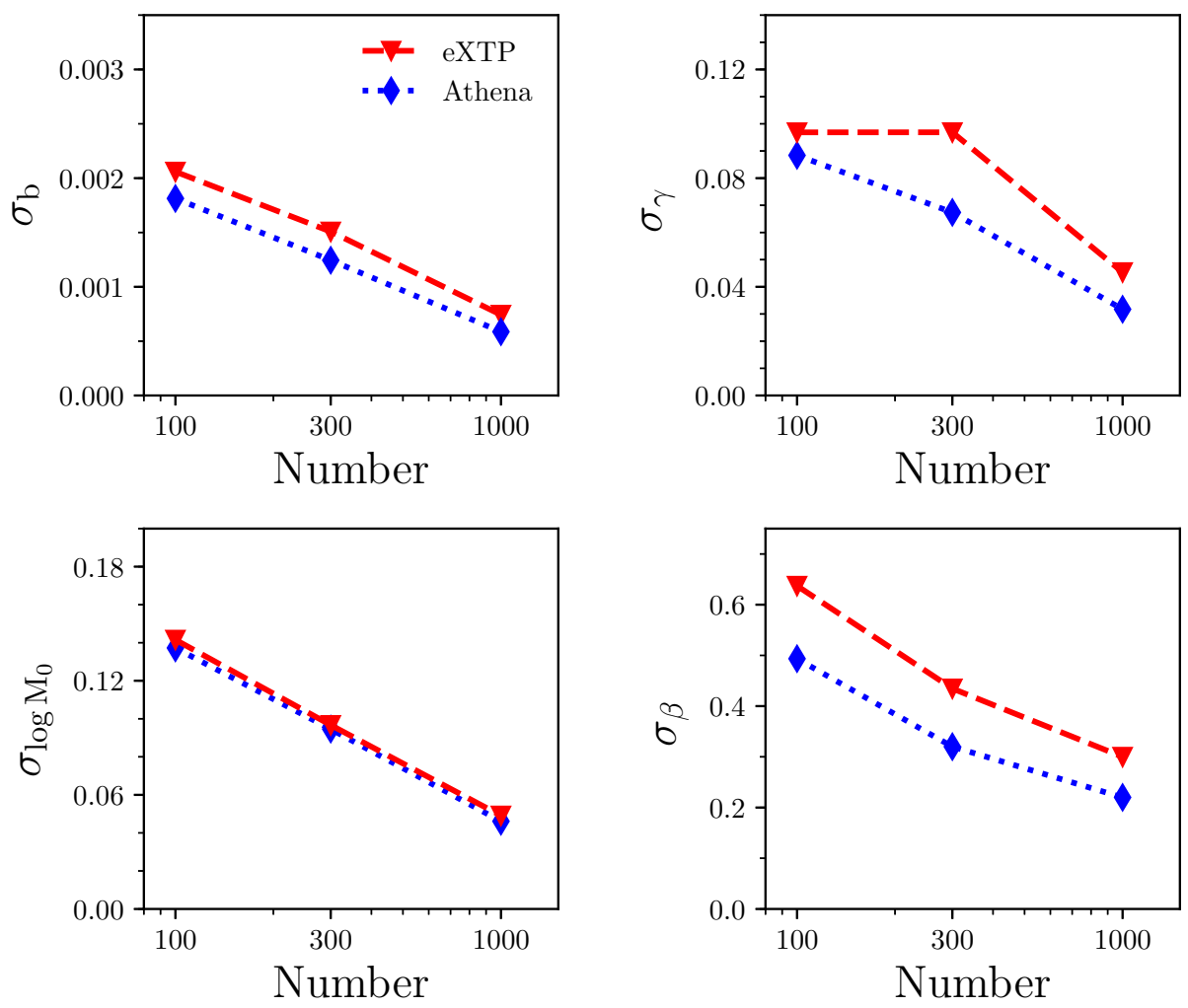

Fig. 9. - Constraint accuracy of the parameters $\left(b, \gamma, M_{0}\right.$, and $\left.\beta\right)$ involved in the accretion Model C, as a function of the size of the mock sample, with assumed spin measurement errors of 0.1 (red triangles) and 0.04 (blue diamonds), roughly corresponding to the capacity of eXTP and Athena, respectively. The symbols are connected with dashed and dotted lines with corresponding colors to guide the eye. The constraint accuracy is quantified by the standard deviation of the MCMC sampling. 
that the accretion model can be well reconstructed if the adopted model is a good approximation to the underlying one. Here we also check whether different accretion models can be distinguished from each other by fitting the mock sample(s) generated from any one of three models in this paper with the other two models.

To compare two competitive models, one may evaluate their relative evidence. The Bayesian evidence is the integration of the likelihood over the whole parameter space, i.e.,

$$
p(D \mid M)=\int p(D \mid M, \boldsymbol{\theta}) p(\boldsymbol{\theta} \mid M) d \boldsymbol{\theta},
$$

where $M$ denotes the model, $p(D \mid M, \boldsymbol{\theta})$ is the likelihood given by Equation (8), and $p(\boldsymbol{\theta} \mid M)$ is the prior of the parameters $\boldsymbol{\theta}$ associated with the model, which is assumed to be a flat distribution. The ratio of Bayesian evidence of two models gives the Bayes factor, i.e., $B_{1,2}=p\left(D \mid M_{1}\right) / p\left(D \mid M_{2}\right)$. We adopt the sample in the upper panel of Figure 3, generated from Model A with $\left(b, \gamma, f_{\mathrm{c}}\right)=(0.012,0.00,0.46)$. The sample includes $100 \mathrm{MBHs}$ with a spin accuracy of 0.1. This is set to check that whether a relatively small sample size and low spin accuracy are able to distinguish between different models, since the results shown in Section 5 indicate that a larger sample with higher spin accuracies will better constrain the model. We then apply Model $\mathrm{C}$ to fit the data by adopting the same Bayesian MCMC procedure. Although with larger uncertainties, the sampling does return peaked posterior distributions with the means and $1 \sigma$ errors of $\left(b, \gamma, m_{0}, \beta\right)=$ $\left(0.020_{-0.004}^{+0.005},-0.14_{-0.10}^{+0.09}, 10.31_{-0.32}^{+0.40}, 1.21_{-0.80}^{+0.89}\right)$.

However, the Bayes factor we find is $B_{A \cdot C}=19.2$, indicating more evidence for the input Model A over Model C.

\subsection{Selection Bias}

Current spin measurement of MBHs relies on the modeling of the reflected X-ray spectra of AGNs, and especially the red wing of the Fe K $\alpha$ line, which might suffer from selection biases toward high signal-to-noise ratios and thus X-ray-luminous sources (e.g., Brenneman 2013). This may lead to a preferred selection of faster-rotating or higher-mass MBHs. In our simulations in Section 5 we do not consider such selection bias, as the number of current spin samples is still quite limited and quantitative description of the selection bias is still uncertain. As a simple consideration, we select mock spin samples according to Model A with $\left(b, \gamma, f_{\mathrm{c}}\right)=(0.005,0.50,0.20)$, like the lower panel of Figure 3 but with a cutoff in bolometric luminosity. With a similar sample size, we add a linear dependence of spin accuracy on the $\mathrm{MBH}$ mass, i.e., 0.1 for $10^{6} M_{\odot}$ MBHs and 0.04 for $10^{10} M_{\odot}$ MBHs. By adopting the same Bayesian procedure, we find that the reconstructed $f_{\mathrm{c}}$ is slightly larger than that without considering selection bias $(0.22 \pm 0.03$ vs. $0.19 \pm 0.03)$. In the future, once the spin measurements increase by a large factor, the selection bias may be estimated with detailed consideration of those systems with/without spin measurements. In principle, with such estimations, one may introduce a weight to the spin sample to consider the selection bias and obtain more accurate constraints on $\mathrm{MBH}$ accretion history.

\subsection{Mergers of $\mathrm{MBHs}$}

Our models do not take MBH mergers into account, since MBHs mainly acquire their masses by means of gas accretion, although some works already involved mergers in their models (e.g., Berti \& Volonteri 2008; Barausse 2012). Relativistic simulations reveal that mergers of two MBHs with comparable masses generally result in a spin value of $0.7-0.9$ (e.g., Berti \& Volonteri 2008; Barausse \& Rezzolla 2009). If a merger occurs in the coherent accretion phase, it may lead to a change of the spin from the quasi-equilibrium value, which is mainly determined by the disk-to-BH mass ratio. Therefore, it may lead to some bias in the constraint on $b$. Because more massive BHs have a higher probability of merging, if a merger event happens during the interval of two episodes of chaotic accretion, it will lead to a jump in the $\mathrm{MBH}$ spin. After that, the spin sharply declines and then oscillates. This may affect the constraint on $f_{\mathrm{c}}$. $\mathrm{MBH}$ mergers should in principle be considered in a more comprehensive cosmological model that involves evolution of both MBHs and host galaxies, but beyond the scope of this paper. We defer it to a future work to study the effect of mergers on the cosmic spin distributions of MBHs.

\section{CONCLUSIONS}

In this paper, we investigate constraints on the growth history of MBHs that may be obtained by the spin measurements from X-ray observations in the future, by performing mock simulations and assuming simple twophase accretion models, i.e., MBHs experience coherent accretion followed by periods of chaotic accretion. The cloud mass in each chaotic episode is assumed to follow a power-law dependence on the MBH mass. The division of the two phases is characterized by parameter $f_{\mathrm{c}}$, for which we consider three cases: (i) constant for all MBHs; (ii) a power-law dependence on the $\mathrm{MBH}$ mass; (iii) a hyperbolic-tangent form of dependence on the MBH mass. Those models are capable of producing various spin distributions of the mock samples including: (i) high spins for low-mass BHs and low spins for highmass BHs; (ii) low spins for low- and high-mass BHs, and high spins for intermediate-mass BHs; (iii) high spins for low- and high-mass BHs, and low spins for intermediatemass BHs.

By performing Bayesian MCMC simulations and adopting the mock samples generated from various accretion models, we find that for the simple model with constant $f_{\mathrm{c}}$ for all MBHs, the accretion history can be reasonably reproduced provided that spins of $100 \mathrm{MBHs}$ are measured with an accuracy of 0.1. However, a larger sample (e.g., 300) is required to remove the tail on the left side of the posterior distribution of parameter $f_{\mathrm{c}}$. Models with an additional dependence of $f_{\mathrm{c}}$ on the $\mathrm{MBH}$ mass are further explored. With a weak power-law form of $f_{\mathrm{c}}$, the model is not so well reconstructed, especially for parameters associated with $f_{\mathrm{c}}$, even a sample of $300 \mathrm{MBHs}$ is provided with a spin accuracy of 0.04 . Instead, the model with a stronger (e.g., hyperbolic-tangent) dependence of $f_{\mathrm{c}}$ on the MBH mass can be well reconstructed provided a sample has similar size and spin accuracy.

The effect of a combination of various sample sizes and spin accuracies is additionally explored and we find that generally either a larger sample size or a higher spin accu- 
racy will place a more precise constraint on the accretion model, and a large sample will somehow compensate for a low spin accuracy and vice versa. Future X-ray missions will provide hundreds of spin measurements with a precision of $\lesssim 0.1-0.2$, and the data are not limited to the local universe (e.g., De Rosa et al. 2019), calling for improvements in the modeling of $\mathrm{MBH}$ growth.

We thank the anonymous referee for their helpful suggestions and comments. $\mathrm{X}$. Zhang gives the delayed thanks to Massimo Dotti for his kindness and patience in replying to the emails and helpful discussions on spin evolution for individual black holes. This work is supported by the National Key Program for Science and Technology Research and Development No. 2017YFA0402600 and No. 2016YFA0400704, the National Natural Science Foundation of China under grants No. 11525312, 11890692, 11873056, 11690024, 11991052 , the Fundamental Research Funds for the Central Universities at Xiamen University under grant 20720190115, and the Strategic Priority Research Program of the Chinese Academy of Science Multi-wave band Gravitational Wave Universe (grant No. XDB23040000). X. Zhang acknowledges the support from the China Postdoctoral Science Foundation (2019M662233).

\section{REFERENCES}

Bandler, S. R., Chervenak, J. A., Datesman, A. M., et al. 2019, JATIS, 5, 021017

Barausse, E. 2012, MNRAS, 423, 2533

Barausse, E., \& Rezzolla, L. 2009,ApJ, 704, 40

Bardeen, J. M., Press, W. H., \& Teukolsky, S. A. 1972, ApJ, 178, 347

Bardeen, J. M., \& Petterson, J. A. 1975, ApJ, 195, 65

Barret, D., \& Cappi, M. 2019, A\&A, 628, 5

Barret, D., Lam Trong, T., den Herder, J.-W., et al. 2018, SPIE, 10699, 106991G

Berti, E., \& Volonteri, M. 2008, ApJ, 684, 822

Brenneman, L. 2013, AcPol, 53, 652

De Rosa, A., Uttley, P., Gou, L., et al. 2019, SCPMA, 62, 029504

Dotti, M., Colpi, M., Pallini, S., et al. 2013, ApJ, 762, 68

Fabian, A. C., Rees, M. J., Stella, L., \& White, N. E. 1989, MNRAS, 238, 729

Gallo, E., \& Sesana, A. 2019, ApJ, 883, 18

García, J. A. 2019, BAAS, 51, 284

Gaskin, J. A., Swartz, D. A., Vikhlinin A., et al. 2019, JATIS, 5, 021001

Häring, N., \& Rix, H.-W. 2004, ApJ, 604, 89

Hopkins, P. F., Hernquist, L., Cox, T. J., et al. 2007, ApJ, 669, 67

King, A. R., Lubow, S. H., Ogilvie, G. I., \& Pringle, J. E. 2005, MNRAS, 363, 49

King, A. R., Pringle, J. E., \& Hofmann, J. A. 2008, MNRAS, 385, 1621

Kozłowski, M., Jaroszyński, M., \& Abramowicz, M. A. 1978, A\&A, 63, 209

Laor, A. 1991, ApJ, 376, 90

Li, Y.-R., Wang, J.-M., Cheng, C., \& Qiu, J. 2015, ApJ, 804, 45

Madsen, K. K., Harrison, F., Broadway, D., et al. 2018, SPIE, 10699, 106996M

Marconi, A., Risaliti, G., Gilli, R., et al. 2004, MNRAS, 351, 169

Miller, J. M. 2007, ARA\&A, 45, 441

Moderski, R., Sikora, M., \& Lasota, J.-P. 1998, MNRAS, 301, 142
Novikov, I. D., \& Thorne, K. S. 1973, in Black holes (Les astres occlus), ed. C. Dewitt \& B. Dewitt (New York: Gordon and Breach), 343

Perego, A., Dotti, M., Colpi, M., \& Volonteri, M. 2009, MNRAS, 399, 2249

Ray, R. S., Arzoumanian, Z., Ballantyne, D., et al. 2019, BAAS, 51,231

Reynolds, C. S. 2014, SSRv, 183, 277

Reynolds, C. S. 2019, Nature Astronomy, 3, 41

Sesana, A., Barausse, E., Dotti, M., Rossi, E. M. 2014, ApJ, 794, 104

Sesana, A., Gair, J., Berti, E., \& Volonteri, M. 2011, Phys. Rev. D, 83, 044036

Shakura, N. I., \& Sunyaev, R. A. 1973, A\&A, 24, 337

Shankar, F., Weinberg, D. H., \& Miralda-Escud Âl', J. 2009, ApJ, 690,20

Shen, Y., Greene, J. E., Strauss, M. A., Richards, G. T., \& Schneider, D. P. 2008, ApJ, 680, 169

Shen, Y., Richards, G. T., Strauss, M. A., et al. 2011, ApJS, 194, 45

Sołtan, A. 1982, MNRAS, 200, 115

Tanaka, Y., Nandra, K., Fabian, A. C., et al. 1995, Nature, 375, 659

Thorne, K. S. 1974, ApJ, 191, 507

Vasudevan, R. V., Fabian, A. C., Reynolds, C. S., et al. 2016, MNRAS, 458, 2012

Volonteri, M., Sikora, M., Lasota, J.-P., \& Merloni, A. 2013, ApJ, 775,94

Yu, Q., \& Lu, Y. 2004, ApJ, 602, 603

Yu, Q., \& Tremaine, S. 2002, MNRAS, 335, 965

Zhang, S., Santangelo, A., Feroci, M., et al. 2019, SCPMA, 62 029502

Zhang, X., \& Lu, Y. 2019, ApJ, 873, 101

Zhang, X., Lu, Y., \& Liu, Z. 2019, ApJ, 877, 143 